\title{
Directed Random Walks on Polytopes with Few Facets
}

\author{
Malte Milatz \\ mmilatz@inf.ethz.ch \\ Department of Computer Science, ETH Zürich
}

Let $P$ be a simple polytope with $n-d=2$, where $d$ is the dimension and $n$ is the number of facets. The graph of such a polytope is also called a grid. It is known that the directed random walk along the edges of $P$ terminates after $O\left(\log ^{2} n\right)$ steps, if the edges are oriented in a (pseudo-)linear fashion. We prove that the same bound holds for the more general unique sink orientations.

\section{Introduction}

Our research is motivated by the simplex algorithm for linear programming. We consider the variation where the algorithm chooses at each step the next position uniformly at random from all improving neighbouring positions; this rule is commonly called RANDOM-EDGE. Its expected runtime on general linear programs can be mildly exponential; cf. Friedmann et al. [2011]. Better bounds can be hoped for if one imposes restrictions on the input. It is intuitively plausible that RANDOM-EDGE should run very fast if the number of constraints (or facets) is very small in relation to the dimension.

Gärtner et al. [2001] analyzed the performance of RANDOM-EDGE on simple polytopes with $n$ facets in dimension $d=n-2$, and obtained the tight bound $O\left(\log ^{2} n\right)$. It is natural to ask inhowfar this bound depends on the geometry of the problem. To this end we consider the setting where the notion of 'improving' is specified not by a linear objective function, but by a unique sink orientation, which is a more general object with a simple combinatorial definition.

Unique sink orientations have been studied in numerous contexts; see e.g. Szabó and Welzl [2001], Gärtner and Schurr [2006], and Gärtner et al. [2008]. They are defined as follows. A sink in a directed graph is a vertex without any outgoing edges. Now, an orientation of the edges of a polytope is a unique sink orientation if every non-empty face of the polytope has a unique sink. The definition is motivated by the fact that every linear orientation (the orientation obtained from a generic linear objective function) is a unique sink orientation (but the converse does not hold).

The purpose of this note is to prove the following theorem.

Theorem 1. Let $n-d=2$. Let $P$ be a simple $d$-dimensional polytope with $n$ facets, endowed with a unique sink orientation. A directed random walk on $P$, starting at an arbitrary vertex, arrives at the sink after an expected number of $O\left(\log ^{2} n\right)$ steps. 


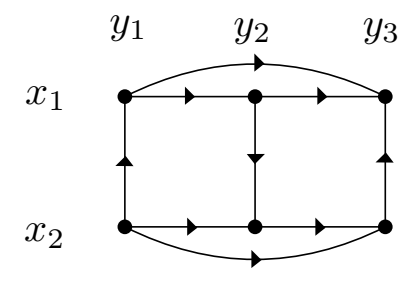

Figure 1: The graph of a polytope (a prism) with $n=5$ facets and dimension $d=3$, which is a grid. Every vertex is identified by a pair $\left\{x_{i}, y_{j}\right\}$. The arrows give an example of a unique sink orientation.

To be perfectly clear, by a directed random walk on a given directed graph we mean the following process: We begin in some given vertex $v_{0}$; we choose one edge uniformly at random from the set of outgoing edges at $v_{0}$; we move to the other endpoint and call it $v_{1}$; then we continue in the same fashion until we possibly arrive at a sink.

For the more restrictive Holt-Klee (or pseudo-linear) orientations, the bound in theorem 1 has been proved by Tschirschnitz [2003]. The general structure of our proof is very similar to Tschirschnitz'; the only notable deviation will be the proof of lemma 4 .

In order to fix some notation, let $H$ be the set of the $n$ halfspaces that define the polytope $P$, and let $V$ be the set of vertices. We will identify every vertex $v \in V$ with the set

$$
\{h \in H: v \text { lies in the interior of } h\},
$$

so that $V$ becomes a subset of the powerset of $H$.

The following fact appeared as lemma 2.1 in Felsner et al. [2005], and may also be seen as a consequence of the existence of Gale diagrams: Assuming that $P$ is a simple polytope with $n-d=2$, there exists a partition $H=X \cup \dot{\cup}$ such that, under the identification of vertices with subsets of $H$ described above, we have

$$
V=\{\{x, y\}: x \in X, y \in Y\} .
$$

Practically speaking, we can thus refer to each vertex by its $X$-coordinate and $Y$-coordinate. Furthermore, a set of vertices forms a face if and only if it is of the form $V \cap 2^{H^{\prime}}$ for some $H^{\prime} \subseteq H$. In particular, two vertices $v, v^{\prime}$ are adjacent if and only if the sets $v, v^{\prime}$ are not disjoint.

The graph of the polytope $P$ is thus isomorphic to the product of two complete graphs, as in fig. 1. Such a graph is also called a grid. Those readers who are not used to unique sink orientations might at this point want to check that the orientation shown in the figure is indeed a unique sink orientation. Note in particular that every row or column in fig. 1 is also a face of $P$, the edges of such a face constitute a complete graph, and they must be oriented in an acyclic fashion.

We write $u \rightarrow v$ for a directed edge from a vertex $u$ to a vertex $v$. A non-empty directed path from $u$ to $v$ is denoted by $u \rightarrow^{+} v$. The outmap $\Phi: V \rightarrow 2^{H}$ specifies the outgoing 
edges at each vertex and is defined by

$$
\Phi(v):=\{h \in H \backslash v: v \rightarrow w \text { for some } w \in V \text { with } h \in w\} .
$$

We also abbreviate

$$
\Phi_{X}(v):=\Phi(v) \cap X \text { and } \Phi_{Y}(v):=\Phi(v) \cap Y .
$$

For example, for the top middle vertex of the grid pictured in fig. 1, we have $\Phi_{X}\left(\left\{x_{1}, y_{2}\right\}\right)=$ $\left\{x_{2}\right\}$ and $\Phi_{Y}\left(\left\{x_{1}, y_{2}\right\}\right)=\left\{y_{3}\right\}$.

\section{Milestones}

Given a vertex $v$, the pair $\left(\left|\Phi_{X}(v)\right|,\left|\Phi_{Y}(v)\right|\right)$ is known as the refined out-degree. From Felsner et al. [2005], Lemma 3.1, we know that for every pair of indices $(i, j)$ with $0 \leq i<|X|-1$ and $0 \leq j<|Y|-1$ there exists a unique vertex with refined out-degree $(i, j)$. We use this property to define the following 'milestones' for our random walk.

The number of milestones will be

$$
L:=1+\left\lfloor\log _{2}(\min \{|X|-1,|Y|-1\})\right\rfloor .
$$

For all $i \in\{1 \ldots L\}$, let $w^{i}$ denote the unique vertex of $P$ with refined out-degree $\left(2^{i-1}, 2^{i-1}\right)$. (The indices are chosen in such a way that the vertex $w^{i}$ exists and has exactly $2^{i}$ outgoing edges.) Furthermore we define $w^{0}$ as the unique sink of $P$; in other words, $w^{0}$ is the unique vertex with refined out-degree $(0,0)$. Now define $W^{i}$ as the set of vertices to which there is a non-empty directed path from this vertex, i.e.,

$$
W^{i}:=\left\{v \in V: w^{i} \rightarrow^{+} v\right\} .
$$

The sets $W^{i}$ serve as a measure of progress: Starting from a vertex in the set $W^{i+1}$, the next 'milestone' is hit when the random walk arrives for the first time in a vertex of $W^{i}$; and once the random walk arrives in some $W^{i}$, it stays therein. Note that the indices are counting down: The random walk arrives in the global sink as soon as the milestone $W^{0}=\left\{w^{0}\right\}$ is hit. Our goal is now to prove the following propositions.

Proposition 1. The expected time until the random walk, starting from a vertex in $W^{i+1}$, arrives in $W^{i}$, is bounded by $O(\log n) .(i=0, \ldots, L-1$.)

Proposition 2. The expected time until the random walk, starting from an arbitrary position, arrives in $W^{L}$, is also bounded by $O(\log n)$.

The bound $O\left(\log ^{2} n\right)$ in theorem 1 will follow by observing that there are $L+1=O(\log n)$ many milestones, each of which is hit - according to the two propositions - after at most $O(\log n)$ steps in expectation.

The technical statements of the following lemma will be useful for deducing the orientation of some edges incident to the current position. The statements are known or follow readily from known results. ${ }^{1}$

\footnotetext{
${ }^{1}$ In particular, parts (a) and (b) of the lemma appear as lemma 4.6 in Tschirschnitz [2003]; part (c) is lemma 4.8 in Tschirschnitz [2003]; part (d) can be proved using (c); and part (e) is from Gärtner et al. [2008].
} 


\section{Lemma 1.}

(a) If a vertex $v$ satisfies $v \subseteq \Phi\left(w^{i}\right)$, then $v \in W^{i}$.

(b) For all $v \in W^{i}, v \cap \Phi\left(w^{i}\right) \neq \emptyset$.

(c) Let $v, w \in V$ and assume $w \not^{+} v$. Then either $\Phi_{X}(v) \supseteq \Phi_{X}(w)$ or $\Phi_{Y}(v) \supseteq \Phi_{Y}(w)$ holds.

(d) $\left|\Phi\left(w^{i+1}\right) \cup \Phi\left(w^{i}\right)\right| \leq 5 \cdot 2^{i-1}$.

(e) Every unique sink orientation of $P$ is acyclic.

\section{Proof of proposition 1}

We write $v_{0}, v_{1}, \ldots$ for the positions of the random walk, where we consider the starting position $v_{0}$ as a fixed element of $V$ (not a random variable). Let $i$ be chosen such that $v_{0} \in W^{i+1}$, and let $T$ denote the hitting time of the set $W^{i}$ :

$$
T=\min \left\{k: v_{k} \in W^{i}\right\} .
$$

We want to bound the expected time until the random walk arrives in $W^{i}$; in other words, we want to bound $\mathrm{E} T$.

One way to look at the directed random walk is as follows: At time $k+1$ it picks a pivot $h_{k+1}$ uniformly at random from the set $\Phi\left(v_{k}\right)$. This pivot determines the edge along which to move away from the vertex $v_{k}$. Concretely, the next position $v_{k+1}$ is the unique neighbour of $v_{k}$ that satisfies $v_{k+1} \subseteq v_{k} \cup\left\{h_{k+1}\right\}$. Note that the pivot $h_{k+1}$ is only defined in this way when the position $v_{k}$ is not already the global sink; so if $v_{k}=w_{0}$ is the global sink then we let $h_{k+1}=\diamond$, where $\diamond$ is just a formal symbol to remind us that the random walk has already terminated.

We define some auxiliary stopping times. Let $\sigma$ denote the first time that an element of the set $\Phi\left(w^{i+1}\right)$ is pivoted. Furthermore let $\tau_{1}<\tau_{2}<\ldots$ be the instants in time when an element of the set $\Phi\left(w^{i+1}\right) \cup \Phi\left(w^{i}\right)$ is pivoted, and let $\tau_{N}$ be the first among these instants when the random walk hits the set $W^{i}$.

More precisely, we let

$$
\begin{aligned}
\sigma & :=\min \left\{k>0: h_{k} \in \Phi\left(w^{i+1}\right) \text { or } h_{k}=\diamond\right\}, \\
\tau_{0} & :=0, \\
\tau_{j} & :=\min \left\{k>\tau_{j-1}: h_{k} \in \Phi\left(w^{i+1}\right) \cup \Phi\left(w^{i}\right) \text { or } h_{k}=\diamond\right\} \quad(j \geq 1), \\
N & :=\min \left\{j: v_{\tau_{j}} \in W^{i}\right\} .
\end{aligned}
$$

We suppress the dependence on $i$ in the notation, considering $i$ (the index of the next milestone) fixed throughout the section.

Lemma 2. The random set $\left\{h_{1}, \ldots, h_{\sigma-1}\right\}$ is always either a subset of $X$ or a subset of $Y$. As a consequence, the vertices $v_{0}, \ldots, v_{\sigma-1}$ share either their $Y$-coordinate or their $X$-coordinate. 
Proof. Assume we encounter the event that, say, $h_{2} \in X$ and $h_{3} \in Y$, where $3<\sigma$. Then $v_{3}=\left\{h_{2}, h_{3}\right\}$. By definition of our stopping time $\sigma$, none of our pivots considered here are elements of $\Phi\left(w^{i+1}\right)$; hence we have $v_{3} \cap \Phi\left(w^{i+1}\right)=\emptyset$. On the other hand, by our choice of $i$ we have $v_{3} \in W^{i+1}$ and thus $v_{3} \cap \Phi\left(w^{i+1}\right) \neq \emptyset$ by lemma $1(\mathrm{~b})$; a contradiction.

Lemma 3. We have $\mathrm{E}[\sigma] \leq \mathrm{H}_{n}+1$, where $\mathrm{H}_{n}$ denotes the $n$th harmonic number.

Proof. By lemma 2, either the pivots $h_{0}, \ldots, h_{\sigma-1}$ are all elements of $X$, or they are all elements of $Y$. Thus it suffices to bound the expected time until, say, the pivot is not an element of $Y$. In terms of fig. 1, this means to bound the expected time until the random walk leaves the current row of the grid. This expectation only becomes larger if we condition on the event that the random walk stays in the current row until it reaches the sink of the row. Reaching the sink of the row can be shown to take $\mathrm{H}_{n}$ steps in expectation; we still have to add 1 for the possible additional step that leaves the current row.

Lemma 4 (Hitting the next milestone). We have $\mathrm{E} T \leq 155\left(\mathrm{H}_{n}+1\right)$.

Proof. We have $T \leq \tau_{N}$; so we will concentrate our efforts on bounding the expectation of $\tau_{N}$. In the following we will need to make the starting position $v_{0}$ of the random walk explicit in the notation; we will do so by writing the starting position as a subscript, as in $\mathrm{E}_{v_{0}}\left[\_\right]$or $\operatorname{Pr}_{v_{0}}\left[\_\right]$.

Using the Markov property we find, for all $j \geq 1$,

$$
\begin{aligned}
\mathrm{E}_{v_{0}}\left[\tau_{j}-\tau_{j-1} \mid j \leq N\right] & =\mathrm{E}_{v_{0}}\left[\tau_{j}-\tau_{j-1} \mid v_{\tau_{j-1}} \notin W^{i}\right] \\
& =\sum_{u \in W^{i+1} \backslash W^{i}} \operatorname{Pr}\left[v_{\tau_{j-1}}=u\right] \mathrm{E}_{v_{0}}\left[\tau_{j}-\tau_{j-1} \mid v_{\tau_{j-1}}=u\right] \\
& =\sum_{u \in W^{i+1} \backslash W^{i}} \operatorname{Pr}_{v_{0}}\left[v_{\tau_{j-1}}=u\right] \mathrm{E}_{u}\left[\tau_{1}\right] \\
& \leq \sup _{u \in W^{i+1}} \mathrm{E}_{u}\left[\tau_{1}\right] \\
& \leq \sup _{u \in W^{i+1}} \mathrm{E}_{u}[\sigma] \\
& \leq \mathrm{H}_{n}+1,
\end{aligned}
$$

where the last step used lemma 3. Hence, applying lemma 5 (appendix) to the sequence $\left(\tau_{j}-\tau_{j-1}\right)_{1 \leq j \leq N}$,

$$
\mathrm{E} T \leq \mathrm{E}\left[\tau_{N}\right]=\mathrm{E}\left[\sum_{j=1}^{N}\left(\tau_{j}-\tau_{j-1}\right)\right] \leq \mathrm{E} N \cdot\left(\mathrm{H}_{n}+1\right) .
$$

It remains to show that the number $\mathrm{E} N$ can be bounded from above by the constant 155 . To this end we consider the following events.

$$
\begin{array}{ll}
\mathcal{E}_{1}: & v_{\tau_{1}} \in W^{i} \text { or } h_{\tau_{1}} \in \Phi\left(w^{i}\right) ; \\
\mathcal{E}_{2}: & v_{\tau_{2}} \in W^{i} \text { or } h_{\tau_{2}} \in \Phi_{\xi}\left(w^{i}\right), \text { where } \xi \in\{X, Y\} \text { such that } h_{\tau_{1}} \notin \xi ; \\
\mathcal{E}_{3}: & v_{\tau_{3}} \in W^{i} .
\end{array}
$$

Claims. For any choice of starting position we have

(i) $\operatorname{Pr}\left[\mathcal{E}_{1}\right] \geq \frac{1}{5}$.

(ii) $\operatorname{Pr}\left[\mathcal{E}_{2} \mid \mathcal{E}_{1}\right] \geq \frac{1}{5}$.

(iii) $\operatorname{Pr}\left[\mathcal{E}_{3} \mid \mathcal{E}_{1}, \mathcal{E}_{2}\right] \geq \frac{1}{5}$. 
Note that the event $\mathcal{E}_{3}$ is equivalent to the event $N \leq 3$ (or in other words, the event that the next milestone is hit no later than at time $\tau_{3}$ ). Thus, once these claims are proved, we can conclude that $\mathrm{E} N$ can be bounded from above by the expected number of steps that it takes a Bernoulli process with parameter $p=\frac{1}{5}$ to hit 3 successive successes. By theorem 2 in the appendix this number is, as desired,

$$
\frac{1-p^{3}}{p^{3}(1-p)}=155
$$

Proofs of the claims.

(i) In order to show (i), it suffices to show $\operatorname{Pr}\left[\mathcal{E}_{1} \mid v_{\tau_{1}} \notin W^{i}\right] \geq \frac{1}{5}$. Let us thus assume $v_{\tau_{1}} \notin W^{i}$, which means that the random walk has not yet hit the next milestone at time $\tau_{1}$. In particular, the random walk has not yet terminated at time $\tau_{1}$. We want to show that now the event $h_{\tau_{1}} \in \Phi\left(w^{i}\right)$ happens with probability at least $\frac{1}{5}$.

By definition of the random walk, the $k$ th pivot $h_{k}$ is chosen uniformly at random from the set of violating constraints, $\Phi\left(v_{k-1}\right)$. This is true for any time $k$ at which the random walk has not yet terminated; now we consider $k=\tau_{1}$ : By definition of $\tau_{1}$, the pivot $h_{\tau_{1}}$ is then chosen (still uniformly at random) only from the smaller set

$$
S:=\Phi\left(v_{\tau_{1}-1}\right) \cap\left(\Phi\left(w^{i+1}\right) \cup \Phi\left(w^{i}\right)\right) .
$$

Since the next milestone has not yet been hit, we know that $v_{\tau_{1}-1} \notin W^{i}$ holds, which is equivalent to writing $w^{i} \nrightarrow^{+} v_{\tau_{1}-1}$. Thus lemma 1(c) implies that either $\Phi_{X}\left(v_{\tau_{1}-1}\right) \supseteq$ $\Phi_{X}\left(w^{i}\right)$ or $\Phi_{Y}\left(v_{\tau_{1}-1}\right) \supseteq \Phi_{Y}\left(w^{i}\right)$ holds. In both these cases we see that $S$ contains a subset of $\Phi\left(w^{i}\right)$ of size $2^{i-1}$. On the other hand,

$$
|S| \leq\left|\Phi\left(w^{i+1}\right) \cup \Phi\left(w^{i}\right)\right| \leq 5 \cdot 2^{i-1}
$$

by lemma 1(d). Hence,

$$
\operatorname{Pr}\left[\mathcal{E}_{1} \mid v_{\tau_{1}} \notin W^{i}\right]=\operatorname{Pr}\left[h_{\tau_{1}} \in \Phi\left(w^{i}\right) \mid v_{\tau_{1}} \notin W^{i}\right] \geq 2^{i-1} /\left(5 \cdot 2^{i-1}\right)=\frac{1}{5} .
$$

(ii) Similarly to how we proceeded in the proof of (i), it suffices to show $\operatorname{Pr}\left[\mathcal{E}_{2} \mid \mathcal{E}_{1}\right.$ and $v_{\tau_{2}} \notin$ $\left.W^{i}\right] \geq \frac{1}{5}$. So we assume $\mathcal{E}_{1}$ and $v_{\tau_{2}} \notin W^{i}$, and without loss of generality we assume $h_{\tau_{1}} \in \Phi_{X}\left(w^{i}\right)$. We want to show that now $h_{\tau_{2}} \in \Phi_{Y}\left(w^{i}\right)$ happens with probability at least $\frac{1}{5}$.

This time the pivot $h_{\tau_{2}}$ is chosen uniformly at random from the set

$$
S^{\prime}:=\Phi\left(v_{\tau_{2}-1}\right) \cap\left(\Phi\left(w^{i+1}\right) \cup \Phi\left(w^{i}\right)\right) .
$$

As before, $\left|S^{\prime}\right| \leq 5 \cdot 2^{i-1}$. Also as before, either $S^{\prime} \supseteq \Phi_{X}\left(w^{i}\right)$ or $S^{\prime} \supseteq \Phi_{Y}\left(w^{i}\right)$ must hold. Now, however, we can observe that the latter alternative must be true (implying the claim because $\left.\left|\Phi_{Y}\left(w^{i}\right)\right|=2^{i-1}\right)$, as follows:

It suffices to show that $h_{\tau_{1}} \notin \Phi\left(v_{\tau_{2}-1}\right)$. (Indeed, this implies $S^{\prime} \nsupseteq \Phi_{X}\left(w^{i}\right)$, which leaves us only with the other alternative, $S^{\prime} \supseteq \Phi_{Y}\left(w^{i}\right)$.) We know from lemma 2 that the 
vertices $v_{\tau_{1}}$ and $v_{\tau_{2}-1}$ share either their $X$-coordinate or their $Y$-coordinate. If they share their $X$-coordinate, so that $h_{\tau_{1}} \in v_{\tau_{2}-1}$, then clearly $h_{\tau_{1}} \notin \Phi\left(v_{\tau_{2}-1}\right)$. If on the other hand our two vertices share their $Y$-coordinate but not their $X$-coordinate, let us consider the grid edge between our two vertices: Since there is a walk from $v_{\tau_{1}}$ to $v_{\tau_{2}-1}$, the edge cannot be directed from $v_{\tau_{2}-1}$ to $v_{\tau_{1}}$, by acyclicity (lemma $1(\mathrm{e})$ ). Note furthermore that we have $v_{\tau_{1}}=\left\{h_{\tau_{1}}, y\right\}$, where $y$ is the shared $Y$-coordinate. Hence the non-existence of a directed edge from $v_{\tau_{2}-1}$ to $v_{\tau_{1}}$ translates into saying that we have $h_{\tau_{1}} \notin \Phi\left(v_{\tau_{2}-1}\right)$, as desired.

(iii) We assume that $\mathcal{E}_{1}$ and $\mathcal{E}_{2}$ occur, and we can also assume $h_{\tau_{1}} \in \Phi_{X}\left(w^{i}\right), h_{\tau_{2}} \in \Phi_{Y}\left(w^{i}\right)$, without loss of generality. We can also assume that the next milestone has not already been hit before the time $\tau_{3}$ (i.e., we assume $v_{\tau_{3}-1} \notin W^{i}$ ). Now we want to show that with probability at least $\frac{1}{5}$ we have $v_{\tau_{3}} \in W^{i}$.

Analogously to the proof of (ii) we note that $h_{\tau_{3}}$ is chosen uniformly at random from a set of cardinality at most $5 \cdot 2^{i-1}$, and $\Phi_{X}\left(w^{i}\right)$ is a subset of this set, with cardinality $2^{i-1}$. This shows already that, with probability at least $\frac{1}{5}, h_{\tau_{3}} \in \Phi_{X}\left(w^{i}\right)$. If we can now show that we have

$$
v_{\tau_{3}-1} \cap \Phi_{Y}\left(w^{i}\right) \neq \emptyset
$$

then the claim will follow (because then, with probability at least $\frac{1}{5}$, we have $v_{\tau_{3}} \subseteq$ $\Phi\left(w^{i}\right)$ and hence $v_{\tau_{3}} \in W^{i}$, cf. lemma 1(a)).

By lemma 2 we know that $v_{\tau_{2}}$ and $v_{\tau_{3}-1}$ share either their $X$-coordinate or their $Y$ coordinate. If they share their $Y$-coordinate then we are done, because then $h_{\tau_{2}} \in$ $v_{\tau_{3}-1} \cap \Phi_{Y}\left(w^{i}\right)$. Assume now that they share their $X$-coordinate but not their $Y$ coordinate; we will now examine the $X$ - and $Y$-coordinates of $v_{\tau_{3}-1}$ separately, and show that neither of them can be an element of the set $\Phi\left(w^{i+1}\right)$, which by lemma lemma 1(b) yields a contradiction to the fact that we have $v_{\tau_{3}-1} \in W^{i+1}$.

- The $Y$-coordinate of $v_{\tau_{3}-1}$ is given by $h_{\tau_{3}-1}$, which by definition of our stopping times is not an element of $\Phi\left(w^{i+1}\right)$.

- Let $x$ denote the $X$-coordinate of $v_{\tau_{3}-1}$. This $x$ is also the $X$-coordinate of $v_{\tau_{2}}$, and also the one of $v_{\tau_{2}-1}$. Suppose we had $x \in \Phi\left(w^{i+1}\right)$ : Then by definition of our stopping times $x$ cannot be the pivot $h_{\tau_{2}-1}$; hence, $v_{\tau_{2}-1}=\left\{x, h_{\tau_{2}-1}\right\}$ and $h_{\tau_{2}-1} \in Y$. By lemma 2 we obtain that $\left\{h_{\tau_{1}+1}, \ldots, h_{\tau_{2}-1}\right\}$ is a subset of $Y$, so that as a consequence $v_{1}$ and $v_{\tau_{2}-1}$ share their $X$-coordinate. But the $X$-coordinate of $v_{1}$ is $h_{\tau_{1}}$; thus we have found $x=h_{\tau_{1}} \in \Phi\left(w^{i}\right)$ and $v_{\tau_{2}}=\left\{x, h_{\tau_{2}}\right\} \subseteq \Phi\left(w^{i}\right)$. By lemma 1 (a) we obtain $v_{\tau_{2}} \in W^{i}$. So the next milestone has already been hit by the time $\tau_{2}$ : a contradiction.

This concludes this section, establishing that the expected time going from one milestone to the next is bounded by $O(\log n)$ (proposition 1). A very similar argumentation can be used to also bound by $O(\log n)$ the expected time until the initial milestone is hit, yielding proposition 2. Theorem 1 now follows by observing that there are $O(\log n)$ many milestones. 


\section{Conclusion}

In this note we have shown that the performance of RANDOM-EDGE on simple $d$-polytopes with $d+2$ facets does not suffer if the improving directions are specified by an arbitrary unique sink orientation.

The exact performance of RANDOM-EDGE on simple $d$-polytopes with $d+k$ facets, where $k \geq 3$ is considered constant, remains an open problem. The question is open even for $k=3$ and with the improving directions specified by a linear objective function.

\section{Appendix}

The lemma and the theorem below, both of elementary nature, are used in section 3 .

Lemma 5. Let $X_{1}, X_{2}, \ldots$ be non-negative random variables, let $N$ be a random variable with values in $\mathbb{N}_{0} \cup\{\infty\}$, and let $X=\sum_{j=1}^{N} X_{j}$. Assume that there is $M>0$ such that for all $j, \mathrm{E}\left[X_{j} \mid N \geq j\right] \leq M$. Then

$$
\mathrm{E} X \leq M \cdot \mathrm{E} N
$$

Proof. Without loss of generality we can assume that $\mathrm{E}\left[X_{j} \mid j>N\right]$ vanishes for all $j$. (If this is not the case then we can just replace each $X_{j}$ by the random variable that equals $X_{j}$ when $j \leq N$, and equals 0 otherwise.) Now we can write the expectation of each individual variable $X_{j}$ as

$$
\mathrm{E} X_{j}=\underbrace{\mathrm{E}\left[X_{j} \mid N \geq j\right]}_{\leq M} \operatorname{Pr}[N \geq j]+\underbrace{\mathrm{E}\left[X_{j} \mid N<j\right]}_{=0} \operatorname{Pr}[N<j] \leq M \operatorname{Pr}[N \geq j]
$$

and conclude using monotone convergence:

$$
\mathrm{E} X=\sum_{j \geq 1} \mathrm{E} X_{j} \leq \sum_{j \geq 1} M \cdot \operatorname{Pr}[N \geq j]=M \cdot \mathrm{E} N .
$$

Theorem 2. For $p \in(0,1)$ and $n \in \mathbb{N}_{0}$, let $\tau(p, n)$ denote the first time that, during a Bernoulli process with parameter $p$, one has encountered $n$ successive successes. Its expectation is given by

$$
\mathrm{E}[\tau(p, n)]=\frac{1-p^{n}}{p^{n}(1-p)} .
$$

Proof. By induction on $n$. The equality clearly holds for $n=0$. For $n \geq 1$ we abbreviate the left-hand side by $t_{n}$ and find the recursion

$$
t_{n}=t_{n-1}+p+(1-p)\left(1+t_{n}\right),
$$

which gives

$$
t_{n}=\frac{1+t_{n-1}}{p}=\frac{1}{p}\left(1+\frac{1-p^{n-1}}{p^{n-1}(1-p)}\right)=\frac{1-p^{n}}{p^{n}(1-p)}
$$




\section{References}

Stefan Felsner, Bernd Gärtner, and Falk Tschirschnitz. Grid orientations, $(d, d+2)$-polytopes, and arrangements of pseudolines. Discrete $\&$ Comput. Geom., 34(3):411-437, 2005.

Oliver Friedmann, Thomas Dueholm Hansen, and Uri Zwick. Subexponential lower bounds for randomized pivoting rules for the simplex algorithm. In Proceedings of the 43rd ACM Symposium on Theory of Computing (STOC), pages 283-292, 2011.

Bernd Gärtner and Ingo Schurr. Linear programming and unique sink orientations. In Proc. 17th Annual ACM-SIAM Symposium on Discrete Algorithms (SODA), pages 749-757, 2006.

Bernd Gärtner, József Solymosi, Falk Tschirschnitz, Pavel Valtr, and Emo Welzl. One line and $n$ points. In Jeffrey Scott Vitter, Paul G. Spirakis, and Mihalis Yannakakis, editors, Proceedings on 33rd Annual ACM Symposium on Theory of Computing, July 6-8, 2001, Heraklion, Crete, Greece, pages 306-315. ACM, 2001.

Bernd Gärtner, Walter D. Morris, Jr., and Leo Rüst. Unique sink orientations of grids. Algorithmica, 51:200-235, 2008.

Tibor Szabó and Emo Welzl. Unique sink orientations of cubes. In Proc. of FOCS 2001, pages 547-555, 2001.

Falk Tschirschnitz. LP-related Properties of Polytopes with Few Facets. PhD thesis, ETH Zürich, 2003. 